\title{
PHASE TRANSITIONS IN DE SITTER SPACE ${ }^{\star}$
}

\author{
B ALLEN \\ Untversity of Cambridge, Department of Appled Mathematics and Theoretical Phystcs, Silver St, \\ Cambridge CB3 9EW, England
}

Received 14 July 1982

(Revised 27 January 1983)

\begin{abstract}
This paper uses zeta-function regularisation to calculate the one-loop functional determinants for fields of any spin in De Sitter space As an example, we investigate the Coleman-Weinberg spontaneous symmetry breaking mechanısm in massless scalar electrodynamics The effective potential is calculated in Landau gauge It depends upon the curvature, and upon the renormalised value of $\xi$ (in $\xi R \phi^{2}$ ) The phase transition will be first or second order, and the critical curvature and mass are found The methods can be applied to any gauge theory
\end{abstract}

\section{Introduction}

Guth [1] has suggested that the early universe went through a period of exponential expansion, during which the cosmological length scale increased by at least exp (64). This solves two important problems in cosmology. It explains how the microwave background radiation can be isotropic. It also explains how the universe came to be so close to the critical density separating indefinite expansion from eventual recollapse. This is why it is so much older than the Planck time $10^{-43}$ seconds, which is the timescale of a radiation dominated universe.

The phase of exponential expansion was caused by the vacuum energy of the Higgs scalar field in a grand unified theory, and during it, the spacetime was described by a De Sitter metric. To provide a consistent model of this process, the vacuum energy of the Higgs field must be calculated in De Sitter space rather than in flat Minkowski space. The purpose of this paper is to show how the vacuum energy of a gauge theory can be calculated in De Sitter space.

The exponential expansion began when the average energy of a particle in the universe was about the energy $M_{\mathrm{X}} \simeq 10^{15} \mathrm{GeV}$ at which the interactions of the GUT models are unified. The spontaneous breakdown of symmetry in a gauge theory is described by an effective potential function shown in fig. 1 . This measures the vacuum energy of the fields, and is a function of the Higgs field $\phi$. When the universe was very hot, the field $\phi$ was fluctuating about a mean value zero $\langle\phi\rangle=0$ and the symmetry $\phi \rightarrow-\phi$ of the GUT lagrangian was unbroken. During this time the vacuum energy density $V(0) \approx M_{\mathrm{X}}^{4}$ acted as a repulsive cosmological term in

* Part of this paper first appeared as a Knıght Prize essay 


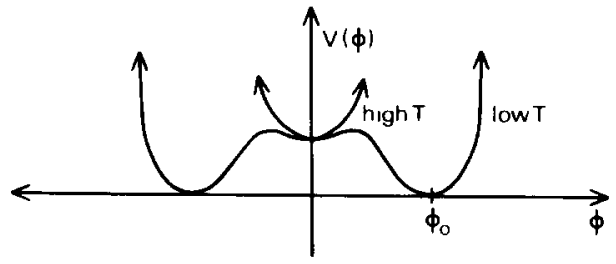

Fig. 1 The effective potential for high and low temperatures. The barrier between $\phi=0$ and $\phi=\phi_{0}$ traps the Higgs field at $\phi=0$ while the universe supercools.

the Einstein equation. It brought about an exponential expansion of the scale factor $\dot{R} / R=H \simeq\left(M_{\mathrm{X}} / M_{\mathrm{P}}\right) M_{\mathrm{X}}$. In these units $\hbar=c=1$ and so $1 \mathrm{GeV} \simeq 10^{-14} \mathrm{~cm}^{-1}$ and $M_{\mathrm{P}} \approx 10^{19} \mathrm{GeV}$ is the Planck mass.

As each comoving volume element of the universe expanded exponentially, the matter cooled, and the potential $V(\phi)$ may have developed new minima away from $\phi=0$. If they became lower than the old minima, the Higgs field $\phi$ would be trapped by the barrier between them, and be unable to make the transition to the new minima at $\phi=\phi_{0}$. As the matter cooled further, the Higgs field eventually approached the broken symmetry phase $\phi=\phi_{0}$. In this phase the vacuum energy of the fields vanished and the period of exponential expansion ceased. The release of the vacuum energy density $V(0)-V\left(\phi_{0}\right)$ created hot matter in the new phase, and the history of the universe then proceeded along like a standard big-bang model.

The outstanding question is how does the phase transition from $\langle\phi\rangle=0$ to $\langle\phi\rangle=\phi_{0}$ take place. Coleman [2] has studied how the phase transition takes place in field theories in flat and curved space. Just as in a phase transition like boiling water, bubbles of the new phase $\langle\phi\rangle=\phi_{0}$ form in the old phase $\langle\phi\rangle=0$. The walls of these bubbles quickly accelerate to lightspeed, and the bubbles grow. However in an exponentially expanding universe, not enough bubbles join together to form a homogeneous universe. The observed universe may have formed from the interior of a single such bubble [3].

A more daring idea [4] is that the phase transition occurs homogeneously. If the effective potential is flat enough at the top of the barrier, a single "bubble" without walls forms everywhere. If this happened, then the big bang is unobservable. The Hawking-Penrose singularity theorems do not apply because they assume an energy condition which is violated in the exponentially expanding phase. The universe we observe began at the moment when the $\phi$ field tunneled out at the maximum of the potential, and the exponential expansion took place while the Higgs field "slid down the hill" to $\phi_{0}$.

If these ideas are correct, then the observed beginning of the universe does not depend upon the initial position and momenta of the matter in it. Any perturbation of the initial matter distribution is exponentially damped, and the observed universe could be thought of as starting, not from an initial singularity, but from an initial De Sitter space containing only the vacuum energy of the fields. 
Each observer in De Sitter space has an event horizon, which he observes radiating a thermal spectrum of particles [5], at a temperature $H / 2 \pi$. This is the lowest equilibrium temperature that any matter can have. So as the matter cools, it approaches this temperature, rather than zero temperature. It is correct to use the flat-space finite-temperature effective potential only when the matter is much hotter than this. When the matter has cooled to this temperature, it is necessary to use an effective potential which is calculated in De Sitter space.

Fortunately there has been a lot of work on quantum field theory in curved space [7]. The path integral formulation seems to be the most appropriate one here, because it is the only technique that takes account of the non-trivial topology of curved space [8]. One difficulty in field theory is that the divergences which result from calculations must be removed by some regularisation technique. The method of dimensional regularisation has been widely used. In this approach one calculates on a spacetime manifold which is $4+\varepsilon$ dimensional, and then cancels the poles which result as $\varepsilon \rightarrow 0$. This technique has been used to study coupling constant renormalisation at several loops [9]. However it is ambiguous because there is no general ansatz for globally continuing a spacetime manifold to $4+\varepsilon$ dimensions. This paper uses zeta function regularisation, which has been developed for doing path integrals in curved space [10]. It is straightforward in the one-loop approximation, but has not been generalised for $N$-loop calculations.

It is possible for the Higgs potential that appears in the GUT lagrangian to lead to symmetry breaking. These potentials are quite complex even though they contain only a few parameters [11]. The other possibility is that the potential for the Higgs field does not directly cause symmetry breaking. Instead, the interactions of the gauge fields with the Higgs field can lead to spontaneous breakdown of symmetry [12]. In curved space this results in very interesting behavior, and it is the most natural way for spontaneous symmetry breaking to occur in a gauge theory.

To calculate the vacuum energy, one must examine the lowest-order quantum corrections to the Higgs potential. These one-loop calculations can be done for any gauge theory. In the second section, we review how they lead to gaussian functional integrals, which can be expressed as functional determinants. In defining the measure of the functional integral, it is necessary to introduce an arbitrary non-zero mass scale $\mu$. The renormalisation group equation ensures that any value of $\mu$ leads to identical physical predictions.

To evaluate the functional determinants, we define them in terms of the generalised zeta function which is the sum of the operator eigenvalues $\zeta(z)=\sum_{n} \lambda_{n}^{-z}$. In highly symmetric spacetimes, the zeta function can be expressed in closed form in terms of the psi function $\psi(z)=(\mathrm{d} / \mathrm{d} z) \log \Gamma(z)$. In the third section, this closed form is found for all spin fields in De Sitter space. This result can be used to determine the one-loop behavior of any gauge theory in De Sitter space.

The simplest gauge theory exhibiting spontaneous symmetry breaking by the Coleman-Weinberg mechanism is scalar electrodynamics. This theory contains 
one real vector field and one complex scalar field, and has a local $U(1)$ gauge symmetry. It is used as an example in sect. 4 to illustrate how the vacuum energy can be calculated from the zeta functions. Shore [13] studied the conformal and minimal cases of this theory using dimensional regularisation. We generalise his results to the arbitrary massless case.

In sect. 5 we give a complete description of how the phase transition takes place as the curvature of the De Sitter space changes. The potential depends upon a single parameter, which we call $P$. It is a combination of $\xi$ and $\lambda / e^{4}$. We prove that for $P>-\frac{1}{6}$ the phase transition is first order, and that for $P<-\frac{1}{6}$ it is second order. We give an exact expression for the critical curvature when $P<-\frac{1}{6}$, and approximate expressions for the critical curvature and mass when $P>2$. Using results from numerical computation, the critical curvature and mass are plotted for $-\frac{1}{6}<P<2$, and the accuracy of the large- $P$ approximation is found. We obtain agreement with the two special cases of Shore [13], who made a small numerical mistake.

In sect. 6 the gravitational part of the action is included. The stationary point is shown to occur for spacetimes much flatter than the generic critical curvature. The extension of these results to the simple SU(5) GUT model will be done in a later paper.

\section{The one-loop approximation}

In the path integral approach to quantum theory, the amplitude is given by an expression [10]

$$
Z=\int \mathrm{d}[g] \mathrm{d}[\phi] \exp \{i I[g, \phi]\},
$$

where $\mathrm{d}[\mathrm{g}]$ is a measure on the space of metrics, $\mathrm{d}[\phi]$ is a measure on the space of fields, and $I[g, \phi]$ is the action. The integral is taken over all fields satisfying given boundary conditions. The dominant contributions come from fluctuations around background fields $\phi_{\mathrm{b}}$ and $g_{\mathrm{b}}$ that extremise the action and satisfy the boundary conditions. The background fields are solutions of the classical field equations.

The action can be expanded in a Taylor series near these classical background fields

$$
I[g, \phi]=I\left[g_{\mathrm{b}}, \phi_{\mathrm{b}}\right]+I_{2}[\tilde{g}]+I_{2}[\tilde{\phi}]+\text { higher order terms , }
$$

where $g=g_{\mathrm{b}}+\tilde{g}$ and $\phi=\phi_{\mathrm{b}}+\tilde{\phi}$. The functionals $I_{2}[\tilde{g}]$ and $I_{2}[\tilde{\phi}]$ are quadratic in $\tilde{g}$ and $\tilde{\phi}$, and the linear terms are absent because $g_{\mathrm{b}}$ and $\phi_{\mathrm{b}}$ satisfy the classical equations of motion. In this paper $g_{b}=$ metric of De Sitter space and $\phi_{b}=$ expectation value of the Higgs field. The one-loop approximation simply neglects 
all the terms of higher order than quadratic. To this order

$$
\log Z=i I\left[g_{\mathrm{b}}, \phi_{\mathrm{b}}\right]+\log \left\{\int \mathrm{d}[\tilde{g}] \exp \left(i I_{2}[\tilde{g}]\right)\right\}+\log \left\{\int \mathrm{d}[\tilde{\phi}] \exp \left(i I_{2}[\tilde{\phi}]\right)\right\} .
$$

In De Sitter space with Lorentz signature $(-,+,+,+)$ the operators appearing $I_{2}$ are unbounded, and so the integrals are ill-defined. To define them, we perform a Wick rotation. In the absence of gravity, this corresponds to replacing Minkowski space with flat euclidean space. In the presence of a background gravitational field, we complexify the manifold and pass into its euclidean $(+,+,+,+)$ section. De Sitter space is a 4-dimensional hyperboloid of constant curvature. It has a unique euclidean section which is a four-sphere $S^{4}$ of radius $a$. The curvature tensors are

$$
R_{\alpha \beta \gamma \delta}=\frac{1}{a^{2}}\left(g_{\alpha \gamma \gamma \beta \delta} g_{\beta \delta \delta} g_{\beta \gamma}\right), \quad R_{\alpha \beta}=\frac{3}{a^{2}} g_{\alpha \beta}, \quad R=\frac{12}{a^{2}} .
$$

On the euclidean section, the operators are bounded below, and the integrals are well-defined. The one-loop expression becomes

$$
\log Z=-S\left[g_{\mathrm{b}}, \phi_{\mathrm{b}}\right]+\log \left\{\int \cdot \mathrm{d}[\tilde{g}] \exp \left(-\boldsymbol{S}_{2}[\tilde{g}]\right)\right\}+\log \left\{\int \mathrm{d}[\tilde{\phi}] \exp \left(-\boldsymbol{S}_{2}[\tilde{\phi}]\right)\right\},
$$

where $S[g, \phi]$ is the euclidean action. Since the four-sphere has no boundary, we integrate over all fluctuations $\tilde{g}$ and $\tilde{\phi}$.

As a simple example, suppose that the euclidean action of a real field $\phi$ is

$$
S[\phi]=\int\left[\frac{1}{2}\left(\partial_{\mu} \phi\right)\left(\partial^{\mu} \phi\right)+V(\phi)\right] \sqrt{g} \mathrm{~d}^{4} x
$$

where $V(\phi)$ is some polynomial function of $\phi$, for instance $V(\phi)=$ $\frac{1}{2}\left(\xi R+m^{2}\right) \phi^{2}+\left(\lambda / 4^{\prime}\right) \phi^{4}$. For constant background fields one can integrate the kinetic term in the action by parts to find

$$
S_{2}[\tilde{\phi}]=\frac{1}{2} \int \tilde{\phi}\left(-\square+V^{\prime \prime}\left(\phi_{b}\right)\right) \tilde{\phi} \mathrm{d} V
$$

where the scalar wave operator is $\square=\nabla^{\mu} \nabla_{\mu}$ and $V^{\prime \prime}\left(\phi_{\mathrm{b}}\right)=\mathrm{d}^{2} V /\left.\mathrm{d} \phi^{2}\right|_{\phi_{\mathrm{b}}}$. By introducing a current $J$, one can ensure that any constant value of $\phi_{\mathrm{b}}$ is a solution of the classical field equations. The effective potential is defined by analogy with the free energy density in thermodynamics. For a constant background field

$$
\begin{gathered}
\exp \left[-\Omega V_{\text {eff }}\left(\phi_{\mathrm{b}}\right)\right] \equiv Z, \\
V_{\text {eff }}\left(\phi_{\mathrm{b}}\right)=V\left(\phi_{\mathrm{b}}\right)-\frac{1}{\Omega} \log \left\{\int \mathrm{d}[\tilde{\phi}] \exp \left(-S_{2}[\tilde{\phi}]\right)\right\},
\end{gathered}
$$

where $\Omega=\int \sqrt{g} \mathrm{~d}^{4} x=\frac{8}{3} \pi^{2} a^{4}$ is the volume of a four-sphere. We have dropped the gravitational terms from $Z$ and will return to them in sect. 6 . 
TABLE 1

Eigenvalue and multiplicity of wave operator for elementary fields in De Sitter space (note $n=0,1,2, \ldots$ )

\begin{tabular}{cccccc}
\hline Spın & Eigenfunction & Condition & Operator & Multiplicity $g_{n}$ & Eigenvalue $\lambda_{n}$ \\
\hline 0 & $\phi_{n}$ & none & $-\nabla_{\mu} \nabla^{\mu}+m^{2}$ & $\frac{1}{6}(n+1)(n+2)(2 n+3)$ & $a^{-2} n(n+3)+m^{2}$ \\
$\frac{1}{2}$ & $\psi_{n}$ & none & $\gamma_{\mu} \partial^{\mu}-m$ & $\frac{2}{3}(n+1)(n+2)(n+3)$ & $\mp_{l} a^{-1}(n+2)-m$ \\
1 & $A_{n}^{\mu}$ & $\nabla_{\mu} A_{n}^{\mu}=0$ & $-\nabla_{\mu} \nabla^{\mu}+m^{2}$ & $\frac{1}{2}(n+1)(n+4)(2 n+5)$ & $a^{-2}\left(n^{2}+5 n+3\right)+m^{2}$ \\
$\frac{3}{2}$ & $\psi_{n}^{\mu}$ & $\nabla_{\mu} \psi_{n}^{\mu}=0$ & $\gamma_{\mu} \partial^{\mu}-m$ & $\frac{4}{3}(n+1)(n+3)(n+5)$ & $\mp l a^{-1}(n+3)-m$ \\
& & $\gamma_{\mu} \psi_{n}^{\mu}=0$ & & & \\
2 & $h_{n}^{\mu \nu}$ & $h_{n}^{\mu \nu}=h_{n}^{\nu \mu}$ & $-\nabla_{\mu} \nabla^{\mu}+m^{2}$ & $\frac{5}{6}(n+1)(n+6)(2 n+7)$ & $a^{-2}\left(n^{2}+7 n+8\right)+m^{2}$ \\
& & $h_{n}{ }^{\nu}{ }^{2}=0$ & & & \\
& & & & \\
\hline
\end{tabular}

We can now express the fluctuations $\tilde{\phi}$ in terms of an orthonormal set of eigenfunctions $\phi_{n}$ of the operator $Q=-\square+V^{\prime \prime}\left(\phi_{\mathrm{b}}\right)$. Each field $\tilde{\phi}$ is a vector in a countable Hilbert space of expansion coefficients. The path integral measure $\mathrm{d}[\phi]$ is taken as a "measure" on this Hilbert space, in the standard way [10]. To make this measure dimensionless, we must introduce a positive quantity $\mu$ with dimensions of $(\text { length })^{-1}=$ mass. We then find that the one-loop effective potential is

$$
V_{1}\left(\phi_{\mathrm{b}}\right)=V\left(\phi_{\mathrm{b}}\right)-\frac{1}{\Omega} \log \left(\operatorname{det} \mu^{-2} Q\left[\phi_{\mathrm{b}}\right]\right)^{-1 / 2} .
$$

The units of this function are mass $/$ length $^{3}=$ mass $^{4}$.

A general gauge theory contains both boson and fermion fields of different spins. These fields can be decomposed in terms of the elementary representations shown in table 1. Gibbons [14] found the eigenvalues and multiplicities of the wave operators for these elementary representations, by considering the action of supersymmetry transformations on product representations of the fields. The one-loop effective potential can be expressed as determinants of these operators, as we have done in the simplest case in (2.9).

We can quickly identify two of the $n=0$ eigenfunctions. The $n=0$ spin- 0 scalar eigenfunction is a constant, and has eigenvalue $m^{2}$. The ten $n=0$ spin-1 transverse vector eigenfunctions are the ten independent Killing vector fields that generate the isometry group $\mathrm{SO}(5)$ of $\mathrm{S}^{4}$. Since they satisfy Killing's equation they are transverse, and by taking a derivative of Killing's equation one can verify that they have eigenvalues $3 / a^{2}+m^{2}$. Our convention in table 1 is that on the euclidean section of the metric, the algebra of the Dirac matrices $\gamma_{\mu}$ is $\left\{\gamma_{\mu}, \gamma_{\nu}\right\}=2 \delta_{\mu \nu}$ and $\gamma_{\mu}^{\dagger}=\gamma_{\mu}$. Any fermionic fields will appear in the lagrangian in terms like $\psi^{4}\left(\gamma_{\mu} \partial^{\mu}-\right.$ $m) \psi$. Because the fermionic fields form an anticommuting Grassman algebra, the gaussian integral leads to a result with a different sign [15] than in (2.9)

$$
Z_{\text {fermon }}=\left(\operatorname{det} \mu^{-2} Q\right)^{1 / 2}
$$

where the field is a Majorana spinor, and $Q$ the operator for spin $-\frac{1}{2}$. 


\section{The zeta function}

Suppose we have an operator $Q$ whose eigenvalues $\lambda_{n}$ each occur $g_{n}$ times. The determinant of $Q$ may be formally written as $\prod_{n}\left(\lambda_{n}\right)^{g_{n}}$ but this infinite product diverges. To give the determinant a sensible meaning, we first define the generalised zeta function [10] of the operator $Q$ by

$$
\zeta(z) \equiv \sum_{n=0}^{\infty} g_{n} \lambda_{n}^{-z}
$$

where $z$ is a complex number. If the $\lambda_{n}$ are eigenvalues of a second-order wave operator, they grow like $n^{2}$ for large $n$. If the spacetime manifold is four-dimensional then the multiplicity $g_{n}$ grows like $n^{3}$ for large $n$. It follows that the sum (3.1) converges for $\operatorname{Re} z>2$. Hence the function defined by (3.1) on the half-plane $\operatorname{Re} z>2$ can be analytically continued to the entire complex plane. The important thing is that $\zeta(z)$ is regular at $z=0$. Its derivative there is formally $\zeta^{\prime}(0)=$ $-\sum_{n} g_{n} \log \lambda_{n}$ and so we define the determinant of $Q$ by

$$
\operatorname{det} Q \equiv \exp \left[-\left.\frac{\mathrm{d}}{\mathrm{d} z} \zeta(z)\right|_{z=0}\right] .
$$

This determinant has some of the properties of an ordinary matrix determinant.

We can easily show that

$$
\operatorname{det}(\mu Q)=\mu^{\zeta(0)} \operatorname{det} Q,
$$

so that $\zeta(0)$ is the number of "rows" of $Q$. If one removes an eigenvalue $\lambda_{0}$ from $Q$ to get $\hat{Q}$ then $\hat{\zeta}(0)=\zeta(0)-1$ and $\zeta^{\prime}(0)=-\log \lambda_{0}+\hat{\zeta}^{\prime}(0)$. Hence if any eigenvalue vanishes, the functional determinant vanishes.

Our task is now to find the zeta function and its derivative at the origin for the operators of table 1 . We can calculate the determinant for a unit radius $(a=1)$ sphere, and use (3.3) to restore the dependence on radius. The fermion eigenvalues of table 1 appear in complex conjugate pairs, which can be multiplied. This "squaring up" converts the first-order operator to a second-order operator. The eigenvalues and multiplicities are then

$$
\begin{gathered}
\lambda_{n}=n^{2}+(2 L+3) n+X, \\
g_{n}=\operatorname{dim}(n+L, L)=\frac{1}{3}(2 L+1)(n+1)\left(n+L+\frac{3}{2}\right)(n+2 L+2),
\end{gathered}
$$

for $n=0,1,2, \ldots$ where the constant $X$ is determined by the mass of the field and by the precise form of the operator in the action. The spin of the field is $L=0$, $\frac{1}{2}, 1, \frac{3}{2}$ or 2 . The multiplicity $g_{n}$ is the dimension of the appropriate representation of the euclidean De Sitter group SO(5). Since the eigenvalues are quadratic in $n$, they may be factored

$$
\lambda_{n}=\left(n+L+\frac{3}{2}+\sqrt{\Delta}\right)\left(n+L+\frac{3}{2}-\sqrt{\Delta}\right),
$$


where

$$
\Delta \equiv\left(L+\frac{3}{2}\right)^{2}-X
$$

The zeta function for a field of spin- $L$ is then

$$
\begin{gathered}
\zeta(z)=\frac{1}{3}(2 L+1) \sum_{n=0}^{\infty}(n+1)\left(n+L+\frac{3}{2}\right)(n+2 L+2) \\
\times\left[\left(n+L+\frac{3}{2}+\sqrt{\Delta}\right)\left(n+L+\frac{3}{2}-\sqrt{\Delta}\right)\right]^{-z}, \\
\zeta(z)=\frac{1}{3}(2 L+1) \sum_{n=L+3 / 2}^{\infty}\left(n^{3}-n\left(L+\frac{1}{2}\right)^{2}\right) n^{-2 z}\left[1-\frac{\Delta}{n^{2}}\right]^{-z} .
\end{gathered}
$$

We now define power series coefficients in the expansion

$$
(1-x)^{-z}=\sum_{k=0}^{\infty} c_{k} x^{k}=1+z x+\frac{1}{2} z(z+1) x^{2}+\frac{1}{6} z(z+1)(z+2) x^{3}+\cdots ;
$$

so we can write our expression for the zeta function as

$$
\zeta(z)=\frac{1}{3}(2 L+1) \sum_{k=0}^{\infty} c_{k} \Delta^{k}\left[\zeta_{\mathrm{R}}\left(2 z+2 k-3, L+\frac{3}{2}\right)-\left(L+\frac{1}{2}\right)^{2} \zeta_{\mathrm{R}}\left(2 z+2 k-1, L+\frac{3}{2}\right)\right] .
$$

The extended Riemann zeta function $\zeta_{\mathbf{R}}(z, \alpha)$ is defined on the half-plane $\operatorname{Re}(z)>1$ by [16]

$$
\zeta_{\mathrm{R}}(z, \alpha) \equiv \sum_{n=0}^{\infty}(n+\alpha)^{-z}
$$

and by analytic continuation elsewhere. The ordinary Riemann zeta function is $\zeta_{\mathrm{R}}(z)=\zeta_{\mathrm{R}}(z, 1) . \zeta_{\mathrm{R}}(z, \alpha)$ is a meromorphic function of $z$ with one pole at $z=1$. Near the pole

$$
\zeta_{\mathrm{R}}(z, \alpha)=\frac{1}{z-1}-\psi(\alpha)+\mathrm{O}(z-1)
$$

where $\psi(z) \equiv(\mathrm{d} / \mathrm{d} z) \log \Gamma(z)$ is the psi (or digamma) function. Consequently the scale dependence is

$$
\zeta(0)=\frac{1}{3}(2 L+1)\left[\zeta_{\mathrm{R}}\left(-3, L+\frac{3}{2}\right)-\left(L+\frac{1}{2}\right)^{2} \zeta_{\mathrm{R}}\left(-1, L+\frac{3}{2}\right)\right]-\frac{1}{3}\left(L+\frac{1}{2}\right)^{3} \Delta+\frac{1}{6}\left(L+\frac{1}{2}\right) \Delta^{2},
$$

and the Riemann zeta function can be expressed as Bernoulli polynomials at negative integer values:

$$
\zeta_{\mathrm{R}}(-1, \alpha)=-\frac{1}{2} \alpha^{2}+\frac{1}{2} \alpha-\frac{1}{12}, \quad \zeta_{\mathrm{R}}(-3, \alpha)=-\frac{1}{4} \alpha^{4}+\frac{1}{2} \alpha^{3}-\frac{1}{4} \alpha^{2}+\frac{1}{120} .
$$

Table 2 gives the values of $\zeta(0)$ obtained from (3.7), (3.14) and (3.15).

To find $\zeta^{\prime}(0)$ we write $\zeta(z)=\zeta(0)+z \zeta^{\prime}(0)+\mathrm{O}\left(z^{2}\right)$ for small $|z|$. Starting with expression (3.11) we use (3.13) on the $k=1$ and $k=2$ terms with $c_{1}=z$ and 
TABLE 2

Values of $\zeta(0)$ for the operators of table 1

\begin{tabular}{ccc}
\hline Spin $=L$ & $\zeta(0)$ in terms of $\Delta$ & $\zeta(0)$ in terms of $X$ \\
\hline 0 & $\frac{1}{12} \Delta^{2}-\frac{1}{24} \Delta-\frac{17}{2880}$ & $\frac{1}{12} X^{2}-\frac{1}{3} X+\frac{29}{90}$ \\
$\frac{1}{2}$ & $\frac{1}{6} \Delta^{2}-\frac{1}{3} \Delta+\frac{11}{180}$ & \\
1 & $\frac{1}{4} \Delta^{2}-\frac{9}{8} \Delta+\frac{863}{960}$ & $\frac{1}{4} X^{2}-2 X+\frac{109}{30}$ \\
$\frac{3}{2}$ & $\frac{1}{3} \Delta^{2}-\frac{8}{3} \Delta+\frac{401}{90}$ & \\
2 & $\frac{5}{12} \Delta^{2}-\frac{125}{24} \Delta+\frac{8383}{576}$ & $\frac{5}{12} X^{2}-5 X+\frac{239}{18}$ \\
\hline
\end{tabular}

$c_{2}=\frac{1}{2} z+\frac{1}{2} z^{2}$. From (3.10) the general $c_{k}=z / k+\mathrm{O}\left(z^{2}\right)$ so we find

$$
\begin{aligned}
\zeta^{\prime}(0)= & \frac{2}{3}(2 L+1)\left[\zeta_{R}^{\prime}\left(-3, L+\frac{3}{2}\right)-\left(L+\frac{1}{2}\right)^{2} \zeta_{R}^{\prime}\left(-1, L+\frac{3}{2}\right)\right]+\frac{1}{12}(2 L+1) \Delta^{2} \\
& +\frac{1}{3}(2 L+1) \sum_{k=1}^{\infty} \frac{\Delta^{k}}{k}\left[\zeta\left(2 k-3, L+\frac{3}{2}\right)-\left(L+\frac{1}{2}\right)^{2} \zeta\left(2 k-1, L+\frac{3}{2}\right)\right],
\end{aligned}
$$

where we have dropped the subscript $\mathrm{R}$ to define $\zeta(1, \alpha) \equiv-\psi(\alpha)$ and $\zeta(n, \alpha) \equiv$ $\zeta_{\mathrm{R}}(n, \alpha)$ for $n$ integer and $n \neq 1$. In (3.16) we indicate the derivative $\zeta_{\mathrm{R}}^{\prime}(z, \alpha) \equiv$ $(\mathrm{d} / \mathrm{d} z) \zeta_{\mathrm{R}}(z, \alpha)$. We can express the sum in (3.16) in terms of the psi function. From [17]

$$
\frac{\mathrm{d}^{n}}{\mathrm{~d} z^{n}} \psi(z)=(-1)^{n+1} n ! \zeta_{\mathrm{R}}(n+1, z), \quad \text { for } n=1,2,3, \ldots,
$$

we can easily show that for complex $z$

$$
\sum_{n=0}^{\infty} \zeta(2 n+1, \alpha) z^{2 n}=-\frac{1}{2}[\psi(\alpha+z)+\psi(\alpha-z)] .
$$

We could sum the series in (3.16) except for the factor of $1 / k$. If we differentiate with respect to $\Delta$ we can write the derivative as

$$
\begin{aligned}
\frac{\mathrm{d}}{\mathrm{d} \Delta} \zeta^{\prime}(0) & =\frac{1}{6}(2 L+1) \Delta+\frac{1}{3}(2 L+1) \zeta_{\mathrm{R}}\left(-1, L+\frac{3}{2}\right) \\
& +\frac{1}{3}(2 L+1)\left(\Delta-\left(L+\frac{1}{2}\right)^{2}\right) \sum_{k=0}^{\infty} \Delta^{k} \zeta\left(2 k+1, L+\frac{3}{2}\right) \\
& =-\frac{1}{6}(2 L+1)\left[\left(\Delta-\left(L+\frac{1}{2}\right)^{2}\right)\left(\psi\left(L+\frac{3}{2}+\sqrt{\Delta}\right)+\psi\left(L+\frac{3}{2}-\sqrt{\Delta}\right)-1\right)+L+\frac{2}{3}\right] .
\end{aligned}
$$

To integrate this function it is convenient to use the shift formula $\psi(1+z)=$ $\psi(z)+1 / z$ to obtain

$$
\frac{\mathrm{d}}{\mathrm{d} \Delta} \zeta^{\prime}(0)=-\frac{1}{6}(2 L+1)\left[\left(\Delta-\left(L+\frac{1}{2}\right)^{2}\right)\left(\psi\left(L+\frac{1}{2}+\sqrt{\Delta}\right)+\psi\left(L+\frac{1}{2}-\sqrt{\Delta}\right)-1\right)-L-\frac{1}{3}\right],
$$


so that

$$
\begin{aligned}
\zeta^{\prime}(0)= & -\frac{1}{3}(2 L+1) \\
& \times\left[\int_{L+1 / 2}^{L+1 / 2+\sqrt{\Delta}} U\left(U-L-\frac{1}{2}\right)(U-2 L-1) \psi(U) \mathrm{d} U\right. \\
& \left.+\int_{L+1 / 2}^{L+1 / 2-\sqrt{\Delta}} U\left(U-L-\frac{1}{2}\right)(U-2 L-1) \psi(U) \mathrm{d} U\right] \\
& +\frac{1}{12}(2 L+1) \Delta^{2}-\frac{1}{6}(2 L+1)\left(L^{2}-\frac{1}{12}\right) \Delta+\frac{2}{3}(2 L+1) \\
& \times\left[\zeta_{\mathrm{R}}^{\prime}\left(-3, L+\frac{3}{2}\right)-\left(L+\frac{1}{2}\right)^{2} \zeta_{\mathrm{R}}^{\prime}\left(-1, L+\frac{3}{2}\right)\right] .
\end{aligned}
$$

The initial value at $\Delta=0$ comes from (3.16) prior to differentiation.

We have found $\zeta(0)$ and $\zeta^{\prime}(0)$ for the operators of table 1 . The $\zeta(0)$ are quadratic functions of $\Delta$ (defined by (3.4) and (3.7)) and are given in table 2 . The functions $\zeta^{\prime}(0)$, given by the integral form in (3.21) have interesting properties, which we will digress briefly to look at.

The integrand of (3.21) has simple poles at $U=-1,-2,-3, \ldots$ The contour of integration is shown in fig. 2. The arrows on the contour show $\Delta$ decreasing. When $\Delta$ is positive, the contour extends along the real axis. It reaches a pole each time $\Delta$ cancels an eigenvalue. Shown in fig. 3 is a graph of the real part of $\zeta^{\prime}(0)$ as a function of $\Delta$. When $\zeta^{\prime}(0) \rightarrow \infty$, the determinant vanishes. The imaginary part of $\zeta^{\prime}(0)$ can be found from $l \pi \times$ (residue of poles) in 3.21 .

Our technique for expressing the determinant in terms of the psi function will work for any quadratic eigenvalue with polynomial multiplicity. For instance, one could calculate the zeta functions for wave operators on $\mathrm{CP}^{2}$ or $\mathrm{S}^{n}$ in the same way. Now, what if the eigenvalue were a polynomial of order $M$, with its multiplicity a polynomial of order $Q$ ?

$$
\lambda_{n}=(n-a)(n-b) \cdots(n-c) .
$$

In theory, we could calculate its determinant $\zeta_{A B}^{\prime} C_{C}(0)$ and scale dependence $\zeta_{A B} C_{C}(0)$ using the zeta function (3.1). We would expect that $\zeta_{A B}^{\prime}{ }_{C}(0)$ is an expression like (3.21), containing one contour integral for each factor in (3.22), whose integrands were polynomials of order $Q$ times psi functions.

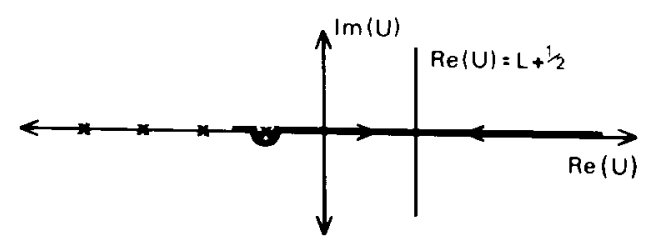

Fig 2 The contour of integration for $\zeta^{\prime}(0)$ in $(3.21)$ If $\Delta>0$ it passes the poles on the real axis If $\Delta<0$ it proceeds up and down the line $\operatorname{Re}(U)=L+\frac{1}{2}$ 


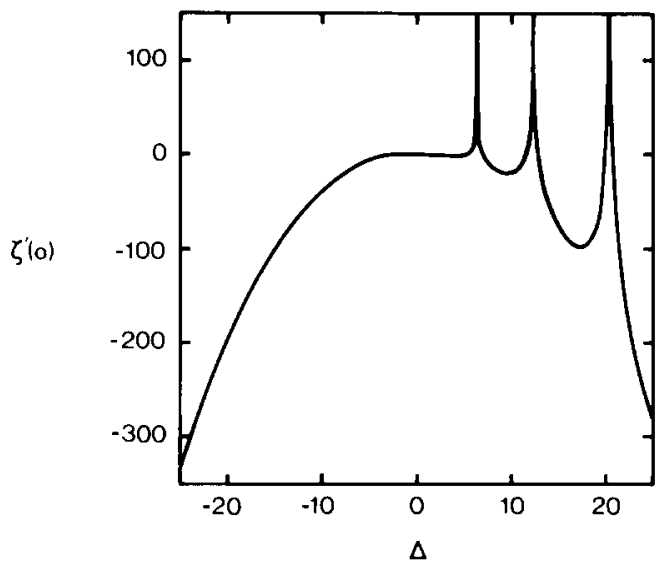

Fig 3 The real part of $\zeta^{\prime}(0)$ for a spin $L=1$ field has logarithmic singularities when $\Delta$ cancels an eıgenvalue The imagınary part is zero for negatıve $\Delta$ and changes discontinuously each time the contour in fig 2 passes by a pole

This suggests another simpler method. Suppose that we use the formula

$$
\operatorname{det}(A B \cdots C)=\operatorname{det} A \operatorname{det} B \cdots \operatorname{det} C,
$$

since each "matrix" $A, B, \ldots, C$ has the same size. Then

$$
\begin{gathered}
\log \operatorname{det}(A B \cdots C)=-\zeta_{A}^{\prime}(0)-\zeta_{B}^{\prime}(0)-\cdots-\zeta_{C}^{\prime}(0), \\
\zeta_{A B} \quad{ }_{C}(0)=\frac{1}{M}\left[\zeta_{A}(0)+\zeta_{B}(0)+\cdots+\zeta_{C}(0)\right],
\end{gathered}
$$

where $A$ is the first factor in (3.22), $B$ is the second factor, and so on. This second method works for the simple case of quadratic polynomials, but has not been proven correct for a general polynomial.

\section{Scalar electrodynamics}

In this section we will calculate the effective potential for scalar electrodynamics. This theory describes a real vector field $A_{\mu}$ and a complex scalar field $\phi$. Its non-negative euclidean action is

$$
S\left[A_{\mu}, \phi\right]=\int \mathscr{L}_{\mathrm{E}} \mathrm{d} V=\int\left[\frac{1}{4} F_{\mu \nu} F^{\mu \nu}+\frac{1}{2}\left(D_{\mu} \phi\right)^{*} D^{\mu} \phi+V(\phi)\right] \mathrm{d} V,
$$

where

$$
\begin{aligned}
F_{\mu \nu} & =\nabla_{\mu} A_{\nu}-\nabla_{\nu} A_{\mu}=\partial_{\mu} A_{\nu}-\partial_{\nu} A_{\mu}, \\
D_{\mu} \phi & =\partial_{\mu} \phi-i e A_{\mu} \phi \\
V(\phi) & =\frac{1}{2}\left(m^{2}+\xi R\right) \phi^{*} \phi+\frac{\lambda}{4 !}\left(\phi^{*} \phi\right)^{2} .
\end{aligned}
$$


The gauge field coupling constant $e$ is the electric charge, the quartic self-interaction coupling constant $\lambda$ is positive, and $\xi$ is the coupling to the curvature. The three numbers $e, \lambda, \xi$ are dimensionless, and the Higgs mass $m^{2}$ has dimensions of length $^{-2}$.

This theory has two kinds of behavior, depending upon whether $\langle\phi\rangle=0$ or $\langle\phi\rangle \neq 0$. In flat space, if $\langle\phi\rangle=0$ then the theory describes a massless transverse vector field (Maxwell field) coupled to a complex charged scalar field. This vector field has two degrees of freedom, and the two real scalar fields have one each. If $\langle\phi\rangle \neq 0$ then the theory describes a massive vector field (with three degrees of freedom) and a single real scalar field which it interacts with. The case $\langle\phi\rangle=0$ is the "unbroken" or high-temperature phase and the case $\langle\phi\rangle \neq 0$ is the "broken symmetry" or low-temperature phase. We will see that the computation of the effective potential proceeds somewhat differently in the two cases.

We first examine the case $\langle\phi\rangle=0$. The complex $\phi$ field can be decomposed into real and imaginary parts

$$
\phi=\varphi_{1}+l \varphi_{2},
$$

where $\varphi_{1}$ and $\varphi_{2}$ are real scalar fields. The vector field can be decomposed with the Hodge decomposition theorem. It says that any differential form is the sum of an exact, a coexact, and a harmonic form. This decomposition is unique, and orthogonal. Hence we can write the 1 -form vector field $A^{\mu}$ as

where

$$
A^{\mu}=A_{\mathrm{T}}^{\mu}+A_{\mathrm{L}}^{\mu}+A_{\mathrm{H}}^{\mu},
$$

$$
\begin{aligned}
\nabla_{\mu} A_{\text {Transverse }}^{\mu} & =0, & & \text { coexact part }, \\
A_{\text {Longtudinal }}^{\mu} & =\partial^{\mu} \chi, & & \text { exact part }, \\
\Delta_{\mu} A_{\text {Harmonic }}^{\nu} & =0, & & \text { harmonic part } .
\end{aligned}
$$

The Hodge-DeRahm operator on 1-forms is

$$
W=\Delta_{\mu \nu}=-g_{\mu \nu} \nabla_{\sigma} \nabla^{\sigma}+R_{\mu \nu},
$$

and $\chi$ in (4.8) is a scalar function. Since the first Betti number of $S^{4}$ is zero there are no harmonic vectors, so $A_{\mathrm{H}}^{\mu}=0$. Since the decomposition is orthogonal

$$
\int A_{\mathrm{T}}^{\mu} A_{\mathrm{L}}^{\nu} g_{\mu \nu} \mathrm{d} V=0
$$

We denote the complete set of fields $B=\left\{A_{\mathrm{T}}, A_{\mathrm{L}}, \varphi_{1}, \varphi_{2}\right\}$.

The action $S[B]$ is invariant under local U(1) gauge transformations. An element $\Omega=\exp (i e \omega(x))$ of the gauge group transforms the fields $B=\{A, \phi\}$ into $B^{\Omega}=$ $\left\{A^{\mu}+\partial^{\mu} \omega(x), \exp (i e \omega(x)) \phi\right\}$ and one can easily show that $S\left[B^{\Omega}\right]=S[B]$.

There is a method due to Faddeev and Popov [18] for extracting the volume of the gauge group from $Z$. Here, with $\langle\phi\rangle=0$ we choose a gauge fixing addition to 
the action

$$
\Delta S[B]=\frac{1}{2} \alpha \int\left(\nabla_{\mu} A^{\mu}\right)^{2} \mathrm{~d} V,
$$

where $\alpha$ is a positive dimensionless number. The Faddeev-Popov determinant $\Delta[B]$ is defined by

$$
\Delta[B] \int \mathrm{d}[\Omega] \exp \left(-\Delta S\left[B^{\Omega}\right]\right)=1,
$$

where $\mathrm{d}[\Omega]$ is a measure on the gauge group. The path integral over all fields $B$ is

$$
Z=\int \mathrm{d}[B] \Delta[B] \exp [-(S[B]+\Delta S[B])] .
$$

In this expression we may regard the Faddeev-Popov determinant $\Delta[B]$ as a jacobian which defines an invariant measure on the space of gauge transformations.

The action $S[B]+\Delta S[B]$ can be expressed in terms of our field decomposition $B=\left\{A_{\mathrm{T}}^{\mu}, A_{\mathrm{L}}^{\mu}, \varphi_{1}, \varphi_{2}\right\}$. In the one-loop approximation, neglecting terms cubic and quartic in the fields

$$
S+\Delta S=\frac{1}{2} \int\left[A_{\mathrm{T}}^{\mu} \Delta_{\mu \nu} A_{\mathrm{T}}^{\nu}+\alpha\left(\nabla_{\mu} A_{\mathrm{L}}^{\mu}\right)^{2}+\varphi_{1}\left(-\square+V^{\prime \prime}(0)\right) \varphi_{1}+\varphi_{2}\left(-\square+V^{\prime \prime}(0)\right) \varphi_{2}\right] \mathrm{d} V,
$$

where $V^{\prime \prime}(0)=m^{2}+\xi R, \square=\nabla_{\mu} \nabla^{\mu}$, and $\Delta_{\mu \nu}=\left(-g_{\mu \nu} \square+R_{\mu \nu}\right)$.

We express our fields $B$ in terms of the basis functions of table 1 .

$$
A_{\mathrm{T}}^{\nu}=\sum_{n=0}^{\infty} a_{n} A_{n}^{\nu}, \quad A_{\mathrm{L}}^{\nu}=\sum_{n=1}^{\infty} b_{n} \partial^{\nu}\left(\frac{\phi_{n}}{\sqrt{\lambda_{n}}}\right), \quad \varphi_{1}=\sum_{n=0}^{\infty} c_{n} \phi_{n}, \quad \varphi_{2}=\sum_{n=0}^{\infty} f_{n} \phi_{n},
$$

where the constants $\left\{a_{n}, b_{n}, c_{n}, f_{n}\right\}$ are the field's coordinates in Hilbert space and have units of length. The basis functions $\partial^{\prime \prime}\left(\phi_{n} / \sqrt{\lambda_{n}}\right)$ are orthonormal and have units of length ${ }^{-2}$. In the Hodge decomposition (4.8) the scalar function $X$ can always be chosen so that $\int \chi \mathrm{d} V=0$. This is why $\phi_{0}$, the constant function, has been omitted.

The functional $\Delta[B]$ defined by (4.13) is independent of the field $B$. Let $\Omega=$ $\exp (i e \omega(x))$ be an element of the gauge group. We can express $\omega(x)$ in terms of the orthonormal scalar eigenfunctions

$$
\omega(x)=\sum_{n=0}^{\infty} \omega_{n} \phi_{n}(x),
$$

where the constants $\omega_{n}$ have units of length ${ }^{2}$ since $\omega(x)$ is dimensionless. The periodicity of the gauge element $\omega \rightarrow \omega+2 \pi / e$ restricts the range of the coefficient $\omega_{0}$. The dimensionless measure on the Lie algebra of $U(1)$ is

$$
\mathrm{d}[\Omega]=\prod_{n=0}^{\infty} \mu^{2} \mathrm{~d} \omega_{n},
$$


where $0 \leqslant \omega_{0} \leqslant\left(\frac{32}{3}\right)^{1 / 2} \pi^{2} e^{-1} a^{2}$ and $-\infty<\omega_{n}<\infty$ for $n \geqslant 1$, and $\mu^{2}$ is a mass ${ }^{2}$. The Faddeev-Popov determinant (4.13) is

$$
\begin{gathered}
\Delta\left[A_{\mathrm{L}}^{\mu}\right] \int \prod_{n=0}^{\infty} \mu^{2} \mathrm{~d} \omega_{n} \exp \left(-\frac{1}{2} \alpha \int\left(\nabla_{\mu}\left(A_{\mathrm{L}}^{\mu}+\partial^{\mu} \omega\right)\right)^{2}\right) \mathrm{d} V=1, \\
\Delta\left[A_{\mathrm{L}}^{\mu}\right] \int \prod_{n=0}^{\infty} \mu^{2} \mathrm{~d} \omega_{n} \exp \left(-\frac{1}{2} \alpha\left(-b_{n}{\sqrt{\lambda_{n}}}^{-} \omega_{n} \lambda_{n}\right)^{2}=1 .\right.
\end{gathered}
$$

From (4.16) $b_{0}=0$, so the functional $\Delta$ is independent of the value of the $b_{n}$ and hence independent of $A^{\mu}$.

$$
\Delta=e \mu^{-2} a^{-2} \operatorname{det}\left(\alpha^{1 / 2} \mu^{-2} \hat{Q}\right) .
$$

The operator $Q=-\square$ on scalars and $\hat{Q}=-\square$ on scalars without the zero mode. We have absorbed the numerical factors of $\pi$ into $\mu$. Defining the path integral measure in (4.14) as

$$
\mathrm{d}[B]=\left(\prod_{n=0} \mu \mathrm{d} a_{n}\right)\left(\prod_{m=1}^{\infty} \mu \mathrm{d} b_{m}\right)\left(\prod_{r=0}^{\infty} \mu \mathrm{d} c_{r}\right)\left(\prod_{p=0}^{\infty} \mu \mathrm{d} f_{p}\right),
$$

we find from (4.15) that

$$
\begin{aligned}
Z_{1}= & \Delta \int \mathrm{d}[B] \exp \left[-\frac{1}{2}\left(\lambda_{n}^{W} a_{n}^{2}+\alpha \lambda_{m}^{O} b_{m}^{2}+\left(\lambda_{r}^{Q}+V^{\prime \prime}(0)\right) c_{r}^{2}+\left(\lambda_{p}^{Q}+V^{\prime \prime}(0)\right) f_{p}^{2}\right)\right], \\
Z_{1}= & e \mu^{-2} a^{-2} \operatorname{det}\left(\alpha^{1 / 2} \mu^{-2} \hat{Q}\right) \operatorname{det}\left(\mu^{-2} W\right)^{-1 / 2} \operatorname{det}\left(\alpha \mu^{-2} \hat{Q}\right)^{-1 / 2} \\
& \times \operatorname{det}\left(\mu^{-2}\left(Q+V^{\prime \prime}(0)\right)\right)^{-1} \\
Z_{1}= & e \mu^{-2} a^{-2} \operatorname{det}\left(\mu^{-2} W\right)^{-1 / 2} \operatorname{det}\left(\mu^{-2} \hat{Q}\right)^{1 / 2} \operatorname{det}\left(\mu^{-2}\left(Q+V^{\prime \prime}(0)\right)\right)^{-1},
\end{aligned}
$$

where $\alpha$ has been cancelled out using (3.3), and we have absorbed the $\pi$ 's into $\mu$. On $\mathrm{S}^{4} R^{\mu \nu}=\left(3 / a^{2}\right) g^{\mu \nu}$ and then from table 1 the operator $W=-g_{\mu \nu} \square+R_{\mu \nu}$ has eigenvalues $a^{-2}\left(n^{2}+5 n+6\right)$. The operator $Q+V^{\prime \prime}(0)$ has eigenvalues $a^{-2}\left(n^{2}+3 n+\right.$ $\left.12 \xi+a^{2} m^{2}\right)$. We will return to $(4.25)$ as soon as we have examined the case $\langle\phi\rangle \neq 0$.

For $\langle\phi\rangle=\phi_{\mathrm{b}}$ the fields can be decomposed into $A^{\mu}=A_{\mathrm{L}}^{\mu}+A_{\mathrm{T}}^{\mu}$ and $\phi=$ $\phi_{\mathrm{b}}+\varphi_{1}+i \varphi_{2}$ where $\varphi_{1}$ and $\varphi_{2}$ are both real scalar functions, and $\phi_{\mathrm{b}}$ is a real constant. The quadratic part of the action (4.1) is

$$
\begin{aligned}
S[B]= & \frac{1}{2} \int\left[A_{\mathrm{T}}^{\mu}\left(\Delta_{\mu \nu}+e^{2} \phi_{\mathrm{b}}^{2} g_{\mu \nu}\right) A_{\mathrm{T}}^{\nu}+\varphi_{1}\left(-\square+V^{\prime \prime}\left(\phi_{\mathrm{b}}\right)\right) \varphi_{1}\right. \\
& \left.+\varphi_{2}\left(-\square+\phi_{\mathrm{b}}^{-1} V^{\prime}\left(\phi_{\mathrm{b}}\right)\right) \varphi_{2}+e^{2} \phi_{\mathrm{b}}^{2} A_{\mathrm{L}}^{\mu} A_{\mathrm{L}}^{\nu} g_{\mu \nu}+2 e \phi_{\mathrm{b}} A_{\mathrm{L}}^{\mu} \partial_{\mu} \varphi_{2}\right] \mathrm{d} V .
\end{aligned}
$$

The potential terms arise from the expansion

$$
V\left(\phi_{\mathrm{b}}+\varphi_{1}+i \varphi_{2}\right)=V\left(\phi_{\mathrm{b}}\right)+\varphi_{1} V^{\prime}\left(\phi_{\mathrm{b}}\right)+\frac{1}{2} \varphi_{1}^{2} V^{\prime \prime}\left(\phi_{\mathrm{b}}\right)+\frac{1}{2} \varphi_{2}^{2} \phi_{\mathrm{b}}^{-1} V^{\prime}\left(\phi_{\mathrm{b}}\right),
$$

of $V$ to second order in $\varphi_{1}$ and $\varphi_{2}$. 
This time we choose the t'Hooft gauge-fixing term

$$
\Delta S[B]=\frac{1}{2} \alpha \int\left[\nabla_{\mu} A^{\mu}-\alpha^{-1} e \phi_{\mathrm{b}} \varphi_{2}\right]^{2} \mathrm{~d} V,
$$

so that the one-loop contribution to the action becomes

$$
\begin{aligned}
S+\Delta S= & \frac{1}{2} \int\left[A_{\mathrm{T}}^{\mu}\left(\Delta_{\mu \nu}+e^{2} \phi_{\mathrm{b}}^{2} g_{\mu \nu}\right) A_{\mathrm{T}}^{\nu}+\alpha A_{\mathrm{L}}^{\mu}\left(-\nabla_{\mu} \nabla_{\nu}+\alpha^{-1} e^{2} \phi_{\mathrm{b}}^{2} g_{\mu \nu}\right) A_{\mathrm{L}}^{\nu}\right. \\
& \left.+\varphi_{1}\left(-\square+V^{\prime \prime}\left(\phi_{\mathrm{b}}\right)\right) \varphi_{1}+\varphi_{2}\left(-\square+\alpha^{-1} e^{2} \phi_{\mathrm{b}}^{2}+\phi_{\mathrm{b}}^{-1} V^{\prime}\left(\phi_{\mathrm{b}}\right)\right) \varphi_{2}\right] \mathrm{d} V .
\end{aligned}
$$

A classical current $J=-V^{\prime}\left(\phi_{\mathrm{b}}\right)$ has been used to eliminate the term linear in $\varphi_{1}$ from the action, making $\phi_{\mathrm{b}}$ a true stationary point.

To one-loop order, the Faddeev-Popov determinant for the gauge-fixing term (4.28) can be written as

$$
\Delta=\left[\int \mu^{2} \mathrm{~d} \omega_{0} \exp \left[-\frac{1}{2} \alpha\left(\alpha^{-1} e^{2} \phi_{\mathrm{b}}^{2}\right)^{2} \omega_{0}^{2}\right]\right]^{-1} \operatorname{det} \alpha^{1 / 2} \mu^{-2}\left(\hat{Q}+\alpha^{-1} e^{2} \phi_{\mathrm{b}}^{2}\right) \text {. }
$$

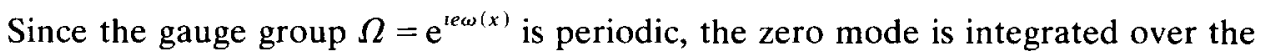
range $0 \leqslant \omega_{0} \leqslant\left(\frac{32}{3}\right)^{1 / 2} \pi^{2} e^{-1} a^{2}$. Dropping the overall numerical factor, we then find

$$
\Delta=\frac{\alpha^{-1 / 2} e^{2} \phi_{\mathrm{b}}^{2} \mu^{-2}}{\operatorname{Erf}\left(\left(\frac{16}{3}\right)^{1 / 2} \pi^{2} \alpha^{-1 / 2} e a^{2} \phi_{\mathrm{b}}^{2}\right)} \operatorname{det} \alpha^{1 / 2} \mu^{-2}\left(\hat{Q}+\alpha^{-1} e^{2} \phi_{\mathrm{b}}^{2}\right),
$$

where the error function is

$$
\operatorname{Erf}(x)=\frac{2}{\sqrt{\pi}} \int_{0}^{x} \exp \left(-y^{2}\right) \mathrm{d} y
$$

For small $|x|$, Erf $(x) \simeq(2 / \sqrt{\pi}) x+\mathrm{O}\left(x^{3}\right)$, so that if $\phi_{\mathrm{b}}=0$, the result (4.31) recovers our previous expression (4.21).

Using the path integral measure (4.22) with the field decomposition (4.16) the path integral now gives

$$
\begin{aligned}
Z_{1}= & \frac{\alpha^{-1 / 2} e^{2} \phi_{\mathrm{b}}^{2} \mu^{-2}}{\operatorname{Erf}\left(\left(\frac{16}{3}\right)^{1 / 2} \pi^{2} \alpha^{-1 / 2} e a^{2} \phi_{\mathrm{b}}^{2}\right)} \operatorname{det} \mu^{-2}\left(\hat{Q}+\alpha^{-1} e^{2} \phi_{\mathrm{b}}^{2}\right)^{1 / 2} \\
& \times \operatorname{det}\left(\mu^{-2}\left(Q+\alpha^{-1} e^{2} \phi_{\mathrm{b}}^{2}+\phi_{\mathrm{b}}^{-1} V^{\prime}\left(\phi_{\mathrm{b}}\right)\right)^{-1 / 2} \operatorname{det}\left(\mu^{-2}\left(Q+V^{\prime \prime}\left(\phi_{\mathrm{b}}\right)\right)\right)^{-1 / 2}\right. \\
& \times \operatorname{det}\left(\mu^{-2}\left(W+e^{2} \phi_{\mathrm{b}}^{2} g_{\mu \nu}\right)\right)^{-1 / 2}
\end{aligned}
$$

The last 2 determinants in (4.33) are gauge-independent and represent the contributions of the real scalar field and the transverse vector field. The other terms depend upon our gauge-fixing parameter $\alpha$, and represent the contributions of the FaddeevPopov factor, longitudinal vector field, and the imaginary scalar field. 
If we specialise to Landau gauge $\alpha \rightarrow \infty$, which was the gauge used in the original flat-space calculations [12], then the path integral yields

$$
\begin{aligned}
Z_{1}= & e \mu^{-2} a^{-2} \operatorname{det}\left(\mu^{-2} \hat{Q}\right)^{1 / 2} \operatorname{det} \mu^{-2}\left(Q+\phi_{\mathrm{b}}^{-1} V^{\prime}\left(\phi_{\mathrm{b}}\right)\right)^{-1 / 2} \\
& \times \operatorname{det} \mu^{-2}\left(Q+V^{\prime \prime}\left(\phi_{\mathrm{b}}\right)\right)^{-1 / 2} \operatorname{det} \mu^{-2}\left(W+e^{2} \phi_{\mathrm{b}}^{2} g_{\mu \nu}\right)^{-1 / 2} .
\end{aligned}
$$

This result agrees with $(4.25)$ in the case $\phi_{\mathrm{b}}=0$, since $V^{\prime \prime}(0)=\lim _{\phi \rightarrow 0} V^{\prime}(\phi) / \phi$. It also yields the correct result for free fields when the gauge coupling constant $e \rightarrow 0$.

Now we introduce notation for the zeta functions evaluated in sect. 3. Let $L=0$, $\frac{1}{2}, 1, \frac{3}{2}, 2$ be spin, and let $\Delta=\left(L+\frac{3}{2}\right)^{2}-X$ where the operator's eigenvalues (3.4) are $\lambda_{n}=n^{2}+(2 L+3) n+X$ for the elementary representations of table 1 . Define $\zeta(L, \Delta)$ as the function in table 2 and $\zeta^{\prime}(L, \Delta)$ as the function (3.21). Shown in fig. 3 is a graph of $\zeta^{\prime}(1, \Delta)$. We can express our determinants in terms of $\zeta$ and $\zeta^{\prime}$. Consider the vector operator $W=\Delta_{\mu \nu}=\left(-g_{\mu \nu} \square+R_{\mu \nu}\right)$. In De Sitter space $R^{\mu \nu}=$ $3 a^{-2} g^{\mu \nu}$, and from table 1 the eigenvalues of $-\square$ on vectors are $a^{-2}\left(n^{2}+5 n+3\right)$, so the eigenvalues of $W$ are $\lambda_{n}^{W}=a^{-2}\left(n^{2}+5 n+6\right)$. Then $\Delta=\frac{25}{4}-6=\frac{1}{4}$. The expression

$\log \operatorname{det} \mu^{-2}\left(W+e^{2} \phi_{\mathrm{b}}^{2} g_{\mu \nu}\right)=-\zeta^{\prime}\left(1, \frac{1}{4}-a^{2} e^{2} \phi_{\mathrm{b}}^{2}\right)-\zeta\left(1, \frac{1}{4}-a^{2} e^{2} \phi_{\mathrm{b}}^{2}\right) \log \left(\mu^{2} a^{2}\right)$,

where we have used (3.3) to get the second term.

We can check the result $(4.25)$ for $Z_{1}$ at $\langle\phi\rangle=0$ by seeing if it gives the correct value of the conformal anomaly. If $\phi_{\mathrm{b}}=0$ and $m^{2}=0$ the classical action and stress-energy vanish, but the one-loop stress-energy is non-zero, because the zero-point fluctuations of the $A$ and $\phi$ field contribute to it. The trace anomaly $[10,13,19]$ is

$$
\int T_{\mu}^{\mu} \mathrm{d} V=-\left.2 a^{2} \frac{\mathrm{d}}{\mathrm{d} a^{2}} \log Z\right|_{\phi_{\mathrm{b}}=0}=-\left.a \frac{\mathrm{d}}{\mathrm{d} a} \log Z\right|_{\phi_{\mathrm{b}}=0},
$$

where $\log Z$ is given by (4.25). We can easily calculate its value from the zeta functions.

$$
\begin{aligned}
\log Z_{1}= & -\log \left(a^{2} \mu^{2}\right)+\frac{1}{2} \zeta\left(1, \frac{1}{4}\right) \log \left(a^{2} \mu^{2}\right)+\frac{1}{2} \zeta^{\prime}\left(1, \frac{1}{4}\right)-\frac{1}{2} \hat{\zeta}\left(0, \frac{9}{4}\right) \log \left(a^{2} \mu^{2}\right) \\
& -\frac{1}{2} \hat{\zeta}^{\prime}\left(0, \frac{9}{4}\right)+\zeta\left(0, \frac{9}{4}-12 \xi\right) \log \left(a^{2} \mu^{2}\right)+\zeta^{\prime}\left(0, \frac{9}{4}-12 \xi\right),
\end{aligned}
$$

where $\hat{\zeta}$ was defined at the beginning of sect. 3 so

$$
\hat{\zeta}\left(0, \frac{9}{4}\right)=\zeta\left(0, \frac{9}{4}\right)-1, \quad \hat{\zeta}^{\prime}\left(0, \frac{9}{4}\right)=\lim _{\varepsilon \rightarrow 0}\left[\zeta^{\prime}\left(0, \frac{9}{4}-\varepsilon\right)+\log \varepsilon\right]
$$

Hence

$$
\int T_{\mu}^{\mu} \mathrm{d} V=-2 a^{2} \frac{\mathrm{d}}{\mathrm{d} a^{2}} \log Z_{1}=-24 \xi^{2}+8 \xi+\frac{4}{90} .
$$

The conformal anomaly at $\xi=0$ is $\frac{4}{90}$, and at $\xi=\frac{1}{6}$ it $1 \mathrm{~s} \frac{64}{90}$, in agreement with other authors $[13,19,20]$. 


\section{The effective potential}

For non-zero $\phi$ the one-loop effective potential is (2.9) $V_{1}(\phi)=$ $V(\phi)-\Omega^{-1} \log Z_{1}$, and using expression (4.34) for the path integral

$$
\begin{aligned}
V_{1}(\phi)= & V(\phi)+\frac{1}{2 \Omega}\left[\log \operatorname{det} \mu^{-2}\left(W+e^{2} \phi^{2} g_{\mu \nu}\right)+\log \operatorname{det} \mu^{-2}\left(Q+V^{\prime \prime}(\phi)\right)\right. \\
& \left.+\log \operatorname{det} \mu^{-2}\left(Q+\phi^{-1} V^{\prime}(\phi)\right)\right],
\end{aligned}
$$

From here on, we drop the "background" subscript from $\phi$. The first determinant comes from the sum of diagrams containing a single closed gauge field loop.

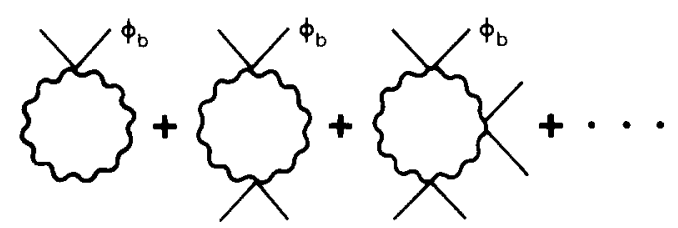

Fig 4 The determinant of the vector operator is the sum of all one gauge-field loop diagrams with "classical field" legs.

The second and third determinant come from the sum of diagrams containing a single closed scalar field loop.

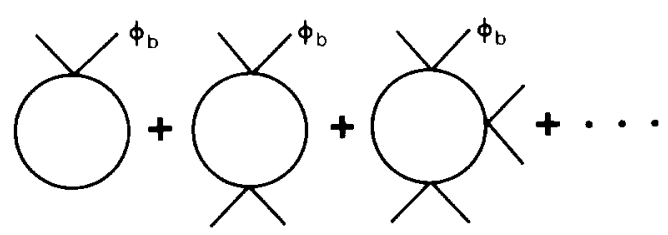

Fig. 5. The determinant of the scalar operator is the sum of all one scalar-field loop diagrams with "classical field" legs.

Each vertex carries a factor of the coupling constant $e^{2}$ or $\lambda$. If $\phi$ were large enough for the second set of diagrams to contribute then the one-loop approximation would break down [12]. We therefore assume that $\lambda$ is of order $e^{4}$ so that the second set of diagrams can be neglected. The vacuum energy then comes only from the fluctuations of the gauge field $A^{\mu}$. The effective potential is then

$$
V_{1}(\phi)=V(\phi)-\frac{1}{2} \Omega^{-1}\left[\zeta^{\prime}\left(1, \frac{1}{4}-a^{2} e^{2} \phi^{2}\right)+\zeta\left(1, \frac{1}{4}-a^{2} e^{2} \phi^{2}\right) \log \left(\mu^{2} a^{2}\right)\right],
$$

where we have used (4.35).

When the radius $a$ of the four-sphere becomes large, the tangent space becomes flat. The large radius limit of $(5.2)$ can be evaluated by finding $\zeta^{\prime}(1, \Delta)$ for large negative $\Delta=\frac{1}{4}-a^{2} e^{2} \phi^{2}$. In this case the contour of integration in (3.21) is along 
the line $\operatorname{Re}(U)=\frac{3}{2}$, and since $\psi(\bar{z})=\overline{\psi(z)}$, if $y^{2}=-\Delta \gg 1$ then

$$
\zeta^{\prime}(1, \Delta) \cong-2 \int_{0}^{y}\left(y^{3}+\frac{9}{4} y\right) \operatorname{Re} \psi\left(\frac{3}{2}+i y\right) \mathrm{d} y+\frac{1}{4} y^{4}+\frac{11}{24} y^{2}+\text { constant } .
$$

For real $y \gg 1$ an asymptotic expansion of the psi function [17]

$$
\operatorname{Re} \psi\left(\frac{3}{2}+i y\right)=\log y+\frac{11}{24} y^{-2}-\frac{127}{960} y^{-4}+\mathrm{O}\left(y^{-6}\right),
$$

can be substituted into (5.3) and yields

$$
\zeta^{\prime}\left(1,-y^{2}\right) \cong-\left(\frac{1}{4} y^{4}+\frac{9}{8} y^{2}+\frac{863}{960}\right) \log y^{2}+\frac{3}{8} y^{4}+\frac{9}{8} y^{2}+\text { constant }
$$

and for large $s^{2}=a^{2} e^{2} \phi^{2}$

$$
\zeta^{\prime}\left(1, \frac{1}{4}-s^{2}\right) \cong-\left(\frac{1}{4} s^{4}+s^{2}+\frac{19}{30}\right) \log s^{2}+\frac{3}{8} s^{4}+s^{2}+\text { constant } .
$$

From table $2, \zeta\left(1, \frac{1}{4}-s^{2}\right)=\frac{1}{4} s^{4}+s^{2}+\frac{19}{30}$ so that for large $a$

$$
\begin{aligned}
V_{1}(\phi)= & V(\phi)+\frac{3 e^{4}}{64 \pi^{2}} \phi^{4}\left[\log \frac{e^{2} \phi^{2}}{\mu^{2}}-\frac{3}{2}\right] \\
& +\frac{3 e^{2}}{16 \pi^{2}} a^{-2} \phi^{2}\left[\log \frac{e^{2} \phi^{2}}{\mu^{2}}-1\right]+\mathrm{O}\left(a^{-4} \log a\right),
\end{aligned}
$$

and as $a \rightarrow \infty$

$$
V_{1}(\phi)=V(\phi)+\frac{3 e^{4}}{64 \pi^{2}} \phi^{4}\left[\log \frac{e^{2} \phi^{2}}{\mu^{2}}-\frac{3}{2}\right] .
$$

We can use this expression to fix the meaning of our constants $e, \lambda$, and $\mu$ in terms of the measurable flat-space values.

Coleman and Weinberg [12] studied the example of massless scalar electrodynamics in flat space. In the large-radius limit of De Sitter space we observe identical behavior. Let $m^{2}=0$, and choose any non-zero values for $\lambda, e, \xi$ and $\mu$. Then the flat space potential (5.8) has a minima at $\phi=\phi_{0}$ where

$$
\phi_{0}^{2}=\frac{\mu^{2}}{e^{2}} \exp \left(1-\frac{8 \pi^{2} \lambda}{9 e^{4}}\right) \text {. }
$$

The experimentally measured mass of the vector boson in flat space is $M=e \phi_{0}$. In terms of this physical parameter, the flat space effective potential (5.8) is

$$
V_{1}(\phi)=\frac{3 e^{4}}{64 \pi^{2}} \phi^{4}\left[\log \frac{e^{2} \phi^{2}}{M^{2}}-\frac{1}{2}\right],
$$

which is identical to the Coleman-Weinberg result. If we replace the parameter $\mu^{2}$ everywhere by $\mu^{2}=M^{2} \exp \left(8 \pi^{2} \lambda / 9 e^{4}-1\right)$, the effective potential will depend upon the ratio $\lambda / e^{4}$ which cannot be determined in flat space. However we will show that an experiment to determine it will actually fix the value of $\xi$. Since $\xi R$ vanishes in flat space, its value cannot be fixed by the large-radius limit. 
We can locate the minima of the potential (5.7) which is a close approximation for $e a \phi \gg 1$. In terms of the dimensionless variable $x=e^{2} \phi^{2} / M^{2}$ we can write the potential as

$$
V_{1}(\phi) \cong \frac{3 M^{4}}{64 \pi^{2}}\left[x^{2}\left(\log x-\frac{1}{2}\right)+A x(\log x+B)\right],
$$

where $A=4 a^{-2} M^{-2}$, and $B=32 \pi^{2} \xi / e^{2}-8 \pi^{2} \lambda / 9 e^{4}$. The minima and maxima of the potential for $x>0$ satisfy $V^{\prime}(\phi)=0$ or

$$
\log x=\frac{-\frac{1}{2} A(B+1)}{x+\frac{1}{2} A}=\frac{-\frac{1}{2} A\left(P+\frac{5}{2}\right)}{x+\frac{1}{2} A},
$$

where we have defined a dimensionless parameter

$$
P \equiv B-\frac{3}{2}=\frac{32 \pi^{2} \xi}{e^{2}}-\frac{8 \pi^{2} \lambda}{9 e^{4}}-\frac{3}{2} .
$$

This equation (5.12) has one, two or three solutions for $x \neq 0$. If $A>2 \exp (-2)$ there is only one solution. As $a \rightarrow \infty, A \rightarrow 0$ and equation (5.12) always has its solution at $x=1$. In other words, as $a \rightarrow \infty$, the minima $\phi_{0}$ of the potential always approaches its flat-space value $e^{2} \phi_{0}^{2}=M^{2}$.

The condition for a phase transition is that two minima in the potential have equal energy. At $x=0$ the potential will be either a maximum or a minimum (we will see which shortly). If it is a minimum then at the critical radius $a_{\mathrm{c}}$ there will be an $x_{\mathrm{c}}>0$ such that $V\left(x_{\mathrm{c}}, a_{\mathrm{c}}\right)=V\left(0, a_{\mathrm{c}}\right)=0$. From (5.11) this means that

$$
\log x=\frac{1}{2}\left[\frac{x-2 A B}{x+A}\right],
$$

and (5.12) will hold at $x_{\mathrm{c}}, a_{\mathrm{c}}$. Eliminating $\log x_{\mathrm{c}}$, and noting that only $A$ depends on the radius, we find that at the critical radius

$$
x_{\mathrm{c}}^{2}-A_{\mathrm{c}}\left(B-\frac{3}{2}\right) x_{\mathrm{c}}+A_{\mathrm{c}}^{2}=0
$$

where $A_{\mathrm{c}}=4 a_{\mathrm{c}}^{-2} M^{-2}$ so $x_{\mathrm{c}}=\frac{1}{2} A_{\mathrm{c}}\left[P \pm\left(P^{2}-4\right)^{1 / 2}\right]$. This means that the measured vector mass $M_{\mathrm{c}}^{2}=M^{2} x_{\mathrm{c}}$ at the phase transition at the critical radius $a_{\mathrm{c}}$ obeys

$$
\frac{1}{2} a_{\mathrm{c}}^{2} M_{\mathrm{c}}^{2}=P+\left(P^{2}-4\right)^{1 / 2},
$$

where we have chosen the positive root, because the other root is a maxima at too small a value of $a e \phi$ for (5.7) to hold. If $P<2$ then approximation (5.7) is no longer valid. However we will show that if $-\frac{1}{6}<P<2$ there is still a first-order phase transition.

We can easily calculate the mass of the vector boson at the phase transition for $P>2$. Since eq. (5.12) holds at the critical radius

$$
\log \frac{M_{c}^{2}}{M^{2}}=\frac{-2\left(P+\frac{5}{2}\right)}{a_{\mathrm{c}}^{2} M_{\mathrm{c}}^{2}+2},
$$




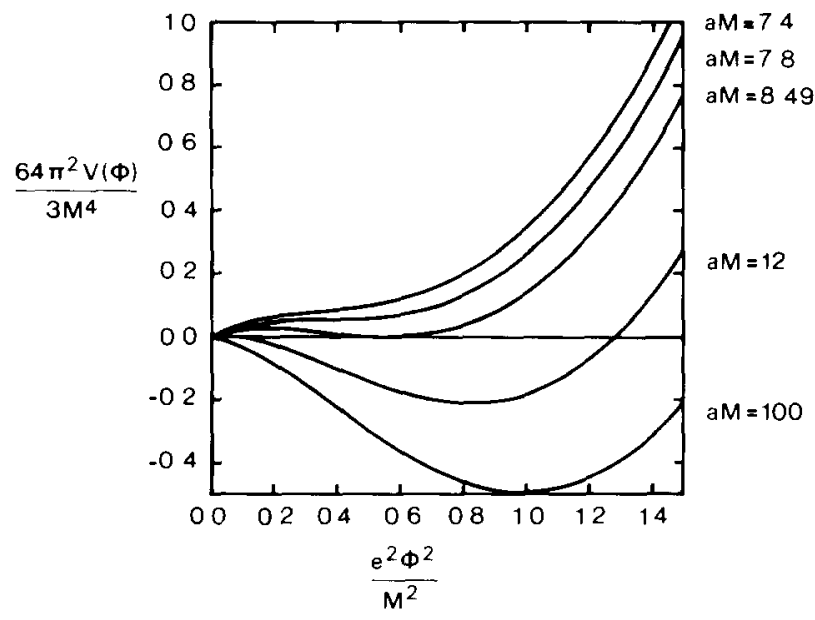

Fig 6 The effective potential for $P=10$, at different radıus' $a$ For $a M=100$ it is indistinguishable from flat space As $a$ decreases, the minima moves up At the critical radius $a_{\mathrm{c}} M=849$ it is at the same height as the minimum at the origın. The critical mass is the value of $e \phi$ at the minimum at the critical radius This phase transition is first order $M$ is the mass of the gauge vector field in flat space

and using (5.16) we find

$$
M_{\mathrm{c}}^{2}=M^{2} \exp \left[\frac{-\left(P+\frac{5}{2}\right)}{1+P+\left(P^{2}-4\right)^{1 / 2}}\right] .
$$

This approximate expression gives the mass of the gauge field at the phase transition in terms of its mass in flat space. Fig. 6 shows a typical potential (5.2) with $P=10$, for values of the radius greater than, equal to, and less than the critical radius $a_{c}$. In fig. 7 the value of the critical mass obtained from (5.18) is compared

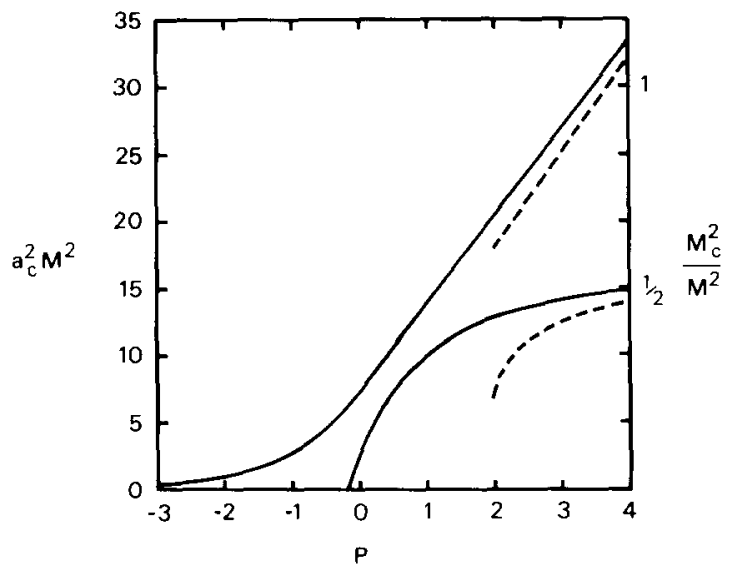

Fig 7 The critical radius and critical mass are functions of $P$ For $P>-\frac{1}{6}$ the phase transition is first order For $P<-\frac{1}{6}$ it is second order The approximate formulas $(518)$ and $(5.19)$ are shown as marked curves They are exact as $P \rightarrow \infty$. 
to the numerically computed value. For $P>4$ the approximation $(5.18)$ is off by $<10 \%$. We can also approximate the critical radius $a_{c}$ in terms of the flat-space vector field mass. Using (5.16) and (5.18) we find

$$
a_{\mathrm{c}}^{2} \cong 2 M^{-2}\left[P+\left(P^{2}-4\right)^{1 / 2}\right] \exp \left[\frac{P+\frac{5}{2}}{1+P+\left(P^{2}-4\right)^{1 / 2}}\right] .
$$

Shore [13] studied the case where $\xi=\frac{1}{6}$ and $e^{2} / 4 \pi$ was small, so that $P \simeq 16 \pi^{2} / 3 e^{2} \gg$ 2. For large $P \gg 2$, the critical radius is $a_{\mathrm{c}}^{2} M^{2}=4 P \exp \left(\frac{1}{2}\right)$ and Shore found

$$
a_{\mathrm{c}}^{2}=\frac{64 \pi^{2}}{3 e^{2}} \exp \left(\frac{1}{2}\right) M^{-2}
$$

in agreement with (5.19).

To see whether $V$ is a minimum or maximum at $\phi=0$ we can expand (5.2) in a power series for small $\phi$. Expression (3.20) for the derivative of $\zeta^{\prime}(0)$ can be easily evaluated at $\Delta=\frac{1}{4}$ and yields

$$
\left.\frac{\mathrm{d}}{\mathrm{d} \Delta} \zeta^{\prime}(1, \Delta)\right|_{\Delta=1 / 4}=\psi(2)+\psi(1)-\frac{1}{3}=-2 \gamma+\frac{2}{3} .
$$

We can take the derivative of (3.20) to find the coefficient of the quartic term

$$
\left.\frac{\mathrm{d}^{2}}{\mathrm{~d} \Delta^{2}} \zeta^{\prime}(1, \Delta)\right|_{\Delta=1 / 4}=-\frac{1}{2}\left[\psi(2)+\psi(1)-2 \psi^{\prime}(2)+2 \psi^{\prime}(1)-1\right]=\gamma-1,
$$

where we have used $\psi(1+z)=\psi(z)+1 / z$ and $\psi^{\prime}(1+z)=\psi^{\prime}(z)-1 / z^{2}$, and Euler's constant $\gamma=-\psi(1) \simeq 0.5772$. So expanding the potential for small $\phi$,

$$
\begin{aligned}
V_{1}(\phi)= & \frac{\text { constant }}{a^{4}}+\frac{3 e^{2}}{16 \pi^{2}}\left[\frac{32 \pi^{2} \xi}{e^{2}}-\frac{8 \pi^{2} \lambda}{9 e^{4}}+\frac{5}{3}-2 \gamma-\log \left(a^{2} M^{2}\right)\right] a^{-2} \phi^{2} \\
& +\frac{3 e^{4}}{64 \pi^{2}}\left[3-2 \gamma-\log \left(a^{2} M^{2}\right)\right] \phi^{4}+\mathrm{O}\left(\phi^{6}\right),
\end{aligned}
$$

where we have used (5.9) to define $M^{2}$ in terms of $\mu^{2}$, and the table 2 expression for $\zeta(1, \Delta)$. The constant in $(5.23)$ is determined by the conformal anomaly $(4.38)$. From (5.23) we can easily see that $V(0)$ is a minimum at the critical radius when $P>2$.

Now suppose that $P<-\frac{1}{6}$. In this case there will be a second-order phase transition when the quadratic term in (5.23) changes sign. The critical radius is given by the exact expression

$$
a_{\mathrm{c}}^{2}=M^{-2} \exp \left(P+\frac{19}{6}-2 \gamma\right) .
$$

At the critical radius the quartic term in (5.23) will be positive, since

$$
\left(3-2 \gamma-\log \left(a_{\mathrm{c}}^{2} M^{2}\right)\right)=3-2 \gamma-\left(P+\frac{19}{6}-2 \gamma\right)=-P-\frac{1}{6}>0 .
$$

In this second-order phase transition, the observed mass of the vector field will 


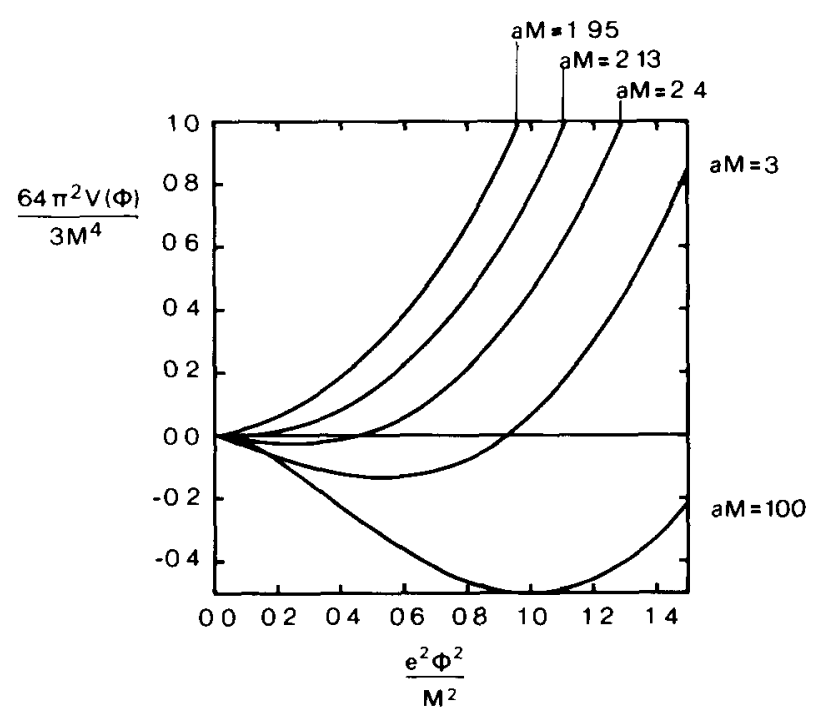

Fig. 8 The effective potential for $P=-\frac{1}{2}$, at different radius' $a$. For $a M=100$ it is indistinguishable from flat space As $a$ decreases, the minima moves up. At the critical radius $a_{\mathrm{c}} M=213$ it reaches the origin This phase transition is second order

vanish at the phase transition, so $M_{\mathrm{c}}=0$. Shown in fig. 8 is the potential for $P=-\frac{1}{2}$.

Shore [13] investigated the case where $\xi=0$ and $8 \pi^{2} \lambda / 9 e^{4}=\log 4 \pi-\gamma+\frac{1}{3}$. In that example $P=-\log 4 \pi+\gamma-\frac{11}{6}<-\frac{1}{6}$, so that there is a second-order phase transition. However we disagree on the critical radius. Shore finds $a_{\mathrm{c}}^{2} M^{2}=$ $\exp \left(-\log 4 \pi-\gamma+\frac{7}{6}\right)$ and we find from $(5.24)$ that $a_{\mathrm{c}}^{2} M^{2}=\exp \left(-\log 4 \pi-\gamma+\frac{4}{3}\right)$. The cause of this small discrepancy is probably a numerical error.

The third possibility occurs when $-\frac{1}{6}<P<2$. For very small radius $a$, both the quadratic and quartic terms in (5.23) are positive. As $a$ increases, the quartic term decreases and vanishes at $\log \left(a^{2} M^{2}\right)=3-2 \gamma$. At this radius the quadratic term is still positive. As $a$ increases more, at $\log \left(a^{2} M^{2}\right)=P+\frac{19}{6}-2 \gamma$ the quadratic term vanishes. The negative quartic term means that there was a first-order phase transition between $3-2 \gamma \leqslant \log \left(a_{\mathrm{c}}^{2} M^{2}\right) \leqslant P+\frac{19}{6}-2 \gamma$. There is no obvious way to find the critical radius analytically.

To calculate it numerically, the potential (5.2) can be written in dimensionless form

$$
\begin{aligned}
\frac{64 \pi^{2} V(\phi)}{3 M^{4}}= & \frac{4}{a^{2} M^{2}}\left[P+\frac{5}{2}-\log \left(a^{2} M^{2}\right)\right] x+\left[1-\log \left(a^{2} M^{2}\right)\right] x^{2} \\
& -\frac{4}{a^{4} M^{4}}\left[\zeta^{\prime}\left(1, \frac{1}{4}-a^{2} M^{2} x\right)-\zeta^{\prime}\left(1, \frac{1}{4}\right)\right],
\end{aligned}
$$

where $x=e^{2} \phi^{2} / M^{2}$ and $a$ has units of $M^{-1}$. The constant term has been chosen 
so that $V(0)=0$, because this has no effect on the critical mass or radius. The resulting values have been plotted in fig. 7 . They were obtained after numerically evaluating $\zeta^{\prime}(1, \Delta)$ which is shown in fig. 3. It might seem natural to give $V(0)$ its conformal anomaly value. However the inflationary universe models which we will now discuss require a different choice.

\section{Adding the gravitational action}

If we neglect gravity, adding a constant $\Lambda$ to the effective potential, so $V(\phi) \rightarrow$ $V(\phi)+\Lambda$, has no effect on the physics. The phase transition radius and critical mass can be determined with $V(0)=0$. However as we stressed, there is a natural value for $V(0)$ given by the conformal anomaly $(4.38)$ which is

$$
V(0)=\frac{3}{16 \pi^{2} a^{4}}\left(-24 \xi^{2}+8 \xi+\frac{4}{90}\right) .
$$

If we include the effects of gravity, this choice is incorrect. The reason is that today's observed cosmological constant is very small.

Presently, we are in the broken symmetry phase with $e \phi_{0}=M$. If $V\left(\phi_{0}\right)=\Lambda$, this uniform background energy density acts like a cosmological constant in the Einstein equation. Today's cosmological constant is very small [21] $\Lambda<10^{-44} M_{\mathrm{X}}^{4}$, so the constant part of $V$ must be determined by the requirement that $V\left(\phi_{0}\right)=0$ in flat space. Even in supersymmetric theories, the vacuum energy does not always vanish (although its divergent part does). But it is forced on us by the observation that $\Lambda$ today is very small.

While the Higgs field is "rolling down the hill", we can ignore the kinetic term in the action, since unless $\left|\phi-\phi_{0}\right| \ll \phi_{0}$, the kinetic term $\left|\left(\partial_{\mu} \phi\right)^{2}\right|$ is of the order $\left(M_{\mathrm{X}} / M_{\mathrm{P}}\right)^{2} V(\phi)$. For a constant background field $\phi$, the effective euclidean action including gravity is

$$
S[\phi, a]=\int\left[-\frac{M_{\mathrm{P}}^{2}}{16 \pi} R+V_{1}(\phi, a)\right] \mathrm{d} V=-2 \pi M_{\mathrm{P}}^{2} a^{2}+\frac{8}{3} \pi^{2} a^{4} V_{1}(\phi, a),
$$

where $M_{\mathrm{P}} \approx 10^{19} \mathrm{GeV}$ is the Planck mass. Demanding that the action is stationary with respect to variations in $a$ and $\phi$ we find $\partial S / \partial a=0$ and $\partial S / \partial \phi=0$. Since $\partial V(\phi, a) / \partial a$ is small, this means that

$$
a^{2}=\frac{3 M_{\mathrm{P}}^{2}}{8 \pi V(\phi, a)}, \quad \frac{\mathrm{d} V(\phi, a)}{\mathrm{d} \phi}=0 .
$$

In flat space, if we set $V\left(\phi_{0}, \infty\right)=0$ then $V(0, \infty)=\left(3 / 128 \pi^{2}\right) M^{4}$.

When the universe had cooled to $K T \simeq M_{\mathrm{X}}$, and $\phi$ was in the symmetric phase, $a=\left(M_{\mathrm{P}} / M_{\mathrm{X}}\right) M_{\mathrm{X}}^{-1}$. Since $M_{\mathrm{P}} / M_{\mathrm{X}} \approx 10^{4}$, this radius was very large compared to $M_{\mathrm{X}}^{-1}$. If $P \ll\left(M_{\mathrm{P}} / M_{\mathrm{X}}\right)^{2}$ then the radius $a \gg a_{\mathrm{c}}$ is much larger than the critical radius, so the potential is very nearly the flat-space potential. 
To solve the horizon and flatness problems with a first-order phase transition requires $P>-\frac{1}{6}$, in a theory which has a vanishing bare scalar mass $m^{2}=0$ in flat space. For $a \simeq\left(M_{\mathrm{P}} / M_{\mathrm{X}}\right) M_{\mathrm{X}}^{-1}$ the $\phi^{4}$ term in (5.23) is negative. There will be a barrier between the two minima of $V(\phi)$ if the $\phi^{2}$ term is positive, so that $P \geqslant 20$. From the definition of $P$ (5.13) this means that if $\lambda$ is of order $e^{4}$ then the phase transition will be first order provided that $\xi \geqslant \alpha$ where $\alpha$ is the coupling constant $\alpha=e^{2} / 4 \pi$. Of course a first-order phase transition is possible for $\xi=0$, provided that $m^{2}>0$.

\section{Conclusion}

Many authors have used massless scalar electrodynamics, to study the phase transition in the early universe. Its effective potential can be easily calculated using zeta functions. Our calculation shows that as in flat space, the ratio $\lambda / e^{4}$ cannot be determined. It simply renormalises $\xi$. The results for the zeta function in sect. 3 can be applied to study any gauge theory at one loop in De Sitter space. We use them in another paper to calculate the effective potential for a basic SU(5) model [22]. They could also be used to study the destabilizing effects of gravitational fluctuations.

Supersymmetric gauge theories are the most interesting ones to study in the inflationary scenario. The vacuum energy that we just calculated was for an ordinary gauge theory containing four boson and no fermion degrees of freedom. It's vacuum energy is actually positive infinite; we calculated its finite part. Supersymmetric theories contain equal numbers of boson and fermion states, and their divergent vacuum energy terms cancel exactly. Hence the remaining finite part is an authentic measure of the vacuum energy [23]. The supersymmetric models which contain spin-2 gravitational fields are known as supergravity theories. They deserve to be closely studied in this context.

The inflationary universe scenario makes very definite predictions: that the universe has close to critical density, and that the angular spectrum of temperature fluctuations in the cosmic background radiation will be scale independent. If the universe turns out to be this way, there may be interesting effects arising from the behavior of field theories in De Sitter space

I would like to thank G.W. Gibbons, S.W. Hawking, and I.G. Moss for their help, and the Marshall Aid Commemoration Commission for a research scholarship.

\section{Note added in proof:}

Part of the speculation at the end of sect. 3 concerning $\log \operatorname{det}(A B \ldots C)$ is incorrect. The r.h.s. of expression (3.24) contains an additional term. This extra term is trivial: it is a polynomial in the coefficients of the (polynomial) eigenvalues 
and multiplicity. This error does not affect any results in the paper, since we do not use the proposed method.

\section{References}

[1] A.H Guth, Phys. Rev D23 (1981) 347

[2] C.G. Callan and S Coleman, Phys. Rev. D15 (1977) 2929, D16 (1977) 1762, S Coleman and F De Lucia, Phys. Rev D21 (1980) 3305

[3] A D Linde, Phys. Lett 108B (1982) 389, A Albrecht and P.J. Steinhardt, Phys. Rev Lett 48 (1982) 1220

[4] S.W. Hawkıng and I G. Moss, Phys Lett 110B (1982) 35

[5] G.W Gibbons and S W Hawking, Phys. Rev D15 (1977) 2738

[6] S Wemberg, Phys Rev D9 (1974) 3357

[7] N D. Birrell and P GW Davies, Quantum fields in curved space (Cambridge Unıversity Press, 1982)

[8] S W Hawkıng, Euclidean quantum gravity, in Recent developments in gravitation Cargese 1978, eds Levy and Deser (Plenum, 1979)

[9] I T Drummond, Phys. Rev. D19 (1979) 1123, I T Drummond and G.M. Shore, Phys Rev. D19 (1979) 1134; Ann of Phys 117 (1979) 89

[10] S W Hawking, Comm Math. Phys 55 (1977) 133

[11] J S Kim, Nucl Phys B196 (1982) 285

[12] S Coleman and E Weinberg, Phys Rev D7 (1973) 1888

[13] G.M Shore, Ann of Phys 128 (1980) 376

[14] S M. Christensen, M J Duff, G W. Gibbons and M. Rocek, One-loop effects in supergravity with a cosmological constant, DAMTP preprint, Cambridge University, 1981

[15] C Itzykson and J.B. Zuber, Quantum field theory (McGraw-Hill, 1980) p. 439

[16] E T Whittaker and G N Watson, A course of modern analysis, (Cambridge University Press, 4th edition, 1952) Chapter 4

[17] M Abramowitz and I Stegun, Handbook of mathematical functions (Dover, 1972) formula 6410

[18] L D Faddeev and V N Popov, Perturbation theory for gauge invariant fields, in Gauge theory of weak and electromagnetıc interactıons, ed C H Lal (World Scientıfic, Singapore, 1981)

[19] M J Duff, Nucl Phys B125 (1977) 334

[20] L S Brown and J P Cassidy, Phys. Rev D15 (1977) 2810

[21] S W Hawkıng, The cosmological constant and the weak anthropic principle, in Proc of the Nuffield Workshop on quantum gravity 1981, eds Isham and Duff (Cambridge University Press, 1982)

[22] B Allen, SU(5) effective potential in De Sitter space, DAMTP preprint, Cambridge University, 1983

[23] B Allen and S Davis, Phys Lett 124B (1983) 363 